\section{Understanding Capgras syndrome}

SIR: In Anderson's comments (Journal, October 1990, 157, 622-623) on Rastogi's article (Journal, June $1990,156,883-884$ ) he says that he is "disappointed to see once again the delusion of inanimate doubles explained away by psychological symbolism". I write to underline this view.

In his letter Dr Anderson also refers to my article (Lipkin, 1988). While I ended this by saying "it is the recommendation of the author that all patients presenting with a Capgras syndrome, whether obviously psychotic or not and whether there be evidence of early dementia or not, be investigated by electrophysiological and brain-imaging techniques", I should like to add a further comment. In my paper I noted that the patient discussed "was referred for second opinion by her consultant psychiatrist ...". It is perhaps not insignificant that the referring consultant psychiatrist was a psychoanalyst. Important aspects of that particular patient's life gave some credence to the notion that her misidentification of her second husband was the manifestation of the psychological mechanism of 'denial'. Psychological treatment proved fruitless (not necessarily because 'denial' was not in operation), because the underlying problem was an organic one.

Therefore I share the view of many others, including $\mathrm{Dr}$ Anderson, that it is necessary to be aware that the Capgras syndrome and Capgras-like phenomena frequently have an organic base and that a preoccupation with putative psychodynamic formulations are not necessarily in the best interests of our patients.

Grovelands Priory Hospital

BRON LIPKIN

The Bourne

Southgate

London N146RA

\section{Reforence}

LIPKIN, B. (1988) Capgras syndrome heralding the development of dementia. British Journal of Psychiatry, 153, 117-118.

\section{Eating disorder in Asian women}

SIR: I read with interest the paper by Dolan et al (Journal, October 1990, 157, 523-528) on the eating attitudes of British women from three different backgrounds (Caucasian, Afro-Caribbean and Asian). Despite their much lower referral rate for psychiatric treatment, Asian women were found to have significantly more disordered eating attitudes which, unlike Caucasian women, were not correlated with a similar degree of anxiety and depression. The paper demonstrated the different relationships between disordered eating attitudes (as shown by the English Eating Attitude Test; EAT) and anxiety and depression, and the implications on clinical presentation of eating disorders. However, a few points deserve mention.

Firstly, the mean age of the Asian sample is high (31.7 years) and not ideal for studying eating attitudes related to acculturation. No information was given as to whether the subjects were immigrants or second-generation British Asians. Previous studies (e.g. Mumford \& Whitehouse, 1988) found disordered eating behaviour to be unexpectedly common in second-generation young British Asians (aged 14 16) who grew up with Western notions of slimness and dieting preoccupations. Social class, a known confounding variable in the EAT, is not mentioned.

Secondly, attitudes toward eating and body shape indeed vary greatly. Anorectic patients from different cultures may manifest different clinical patterns (Lee, 1989), and the use of Western instruments to assess eating attitude and body image in nonWestern groups is fraught with controversies. More than $10 \%$ of the Asians did not attempt the questionnaire because of difficulty in reading English, and one wonders how many of the rest (43 of them, who constituted only $8.3 \%$ of the total sample and were understandably 'precious' subjects) had linguistic and conceptual problems (their education was not mentioned) which might lead to misinterpretations of the EAT and anomalous answers (King \& Bhugra, 1989). This is especially relevant as Asian subjects did not have much anxiety and depression despite high EAT scores. The EAT has been factor-analysed to yield three subscales which may predict clinical patterns and outcome of eating disorders (Garner et al, 1982). It may be instructive for the authors to analyse accordingly for any meaningful (or chaotic) patterns instead of using the global score alone.

Thirdly, the lack of association of disordered eating attitudes with depression paralleled a recent Taiwan study of Chinese patients with eating disorders where, in contrast to Western findings, not one case of major depression was identified (Tseng et al, 1989). But the authors' assertion that "some degree of emotional distress is necessary to precipitate a request for help" is only right with respect to mental health professionals, as Chinese anorectic patients may seek help from physicians (for bloating and emaciation), gynaecologists (for amenorrhoea) or even herbalists (for energy imbalance). They rightly commented that doctors might not recognise eating disorders in non-white women, because the diagnosis of eating disorder involves the dialectical 